\title{
AN ISOMETRIC MAPPING BASED CO-LOCATION DECISION TREE ALGORITHM
}

\author{
Guoqing Zhou ${ }^{1,2}$, Jiandong Wei ${ }^{2,3}$, Xiang Zhou ${ }^{1,2,3, *}$, Rongting Zhang ${ }^{2}$, WeiHuang ${ }^{2,3}$, HongjunSha ${ }^{2}$, JinlongChen ${ }^{2,3}$ \\ 1 School of Microelectronics, Tianjin University, No. 92 W eijin Road, Tianjin 300072, China - zqx0711@tju.edu.cn \\ 2 Guangxi Key Laboratory of Spatial Information and Geomatics, Guilin University of Technology, No. 12 Jian'gan Road, Guilin, \\ Guangxi 541004, China - gzhou@glut.edu.cn \\ 3 Department of Mechanical and Control Engineering, Guilin University of Technology, No. 12 Jian'gan Road, Guilin, Guangxi \\ 541004, China-102016477@glut.edu.cn
}

\section{Commission III, WG III/1}

KEY WORDS: Decision tree (DT), Co-location (Cl), Data mining, Isometric mapping (Isomap), Geodetic distances, Algorithm

\begin{abstract}
:
Decision tree (DT) induction has been widely used in different pattern classification. However, most traditional DTs have the disadvantage that they consider only non-spatial attributes (ie, spectral information) as a result of classifying pixels, which can result in objects being misclassified. Therefore, some researchers have proposed a co-location decision tree (Cl-DT) method, which combines co-location and decision tree to solve the above the above-mentioned traditional decision tree problems. Cl-DT overcomes the shortcomings of the existing DT algorithms, which create a node for each value of a given attribute, which has a higher accuracy than the existing decision tree approach. However, for non-linearly distributed data instances, the euclidean distance between instances does not reflect the true positional relationship between them. In order to overcome these shortcomings, this paper proposes an isometric mapping method based on Cl-DT (called, (Isomap-based Cl-DT), which is a method that combines heterogeneous and Cl-DT together. Because isometric mapping methods use geodetic distances instead of Euclidean distances between non-linearly distributed instances, the true distance between instances can be reflected. The experimental results and several comparative analyzes show that: (1) The extraction method of exposed carbonate rocks is of high accuracy. (2) The proposed method has many advantages, because the total number of nodes, the number of leaf nodes and the number of nodes are greatly reduced compared to Cl-DT. Therefore, the Isomap -based Cl-DT algorithm can construct a more accurate and faster decision tree.
\end{abstract}

\section{INSTRUCTIONS}

In the current researches on remote sensing image classification and information extraction, it is a key issue to meet certain classification accuracy based on multiple categories. In general, according to whether there is a priori knowledge involved in the classification process, the computer automatic classification method is divided into unsupervised classification method and supervision classification method. Decision tree is a basic classification and regression method. It deduces the decision rule set from the disorganized training sample set, and the decision rule set continues to classify the new data. His advantage is the high accuracy of processing high-dimensional data without the need for knowledge or setting of parameters in other areas. But there are some weaknesses in itself: sometimes intelligence does not satisfy people's expectations and there are more tree nodes and hierarchies. Another defect is also the problem to be solved in this paper. The traditional decision tree does not consider the spatial relationship between attributes. Such as the space-time domain in space-time relationship. Therefore, some researchers have proposed a joint position decision tree (Cl-DT) method in order to solve the shortcomings of the above traditional decision tree(Zhou,2011,2012 and 2016). Cl-DT overcomes the disadvantages of the existing DT algorithm, which creates a node for each value of a given attribute. The Cl-DT is more accurate than the existing decision tree method. However, for non-linearly distributed data instances, the Euclidean distance between instances does not reflect the true positional relationship between them. The reason for this is as follows: when determining the distance between two points in an area, the distance between two points is considered without considering the connectivity of the area, only the abstract distance between the starting point and the ending point is considered. In order to overcome the limitation of the Euclidean distance, the above problems are satisfactorily solved. In the actual analysis and application process, we introduce the concept of geodetic distance in the field of mathematical morphology into the real field of spatial analysis ( Frank, 2000). In order to overcome these shortcomings, a new method is proposed in this paper, that is a method called Isometric Mapping (Isomap)-based Cl-DT, which combines Isomap with Cl-DT. First, construct a neighborhood connection diagram for each input data point. Next, the shortest path in the adjacency graph is used to obtain the approximate geodesic distance instead of the classical Euclidean distance, which cannot represent the internal polymorphic structure. So the method of ranging mapping uses the geodetic distance instead of the Euclidean distance between the non-linearly distributed instances, which can reflect the true distance between the instances.

\section{The Algorithm of Isometric Mapping (ISOMAP)}

\subsection{Definition}

Isomap is a linear mapping method, and this can be seen as Metric multidimensional deformation (K. Weinberger, 2006). In this method, the data can be expanded so that the Euclidean

\footnotetext{
* Corresponding author: Xiang Zhou; Email: zqx0711@ glut.edu.cn
} 
distance between the original instances can be approximately preserved in the low-dimensional manifold space. The Isomap algorithm is utilized to unfold the input data, and the geodesic distance is calculated.

\subsection{The Algorithm of Isomap}

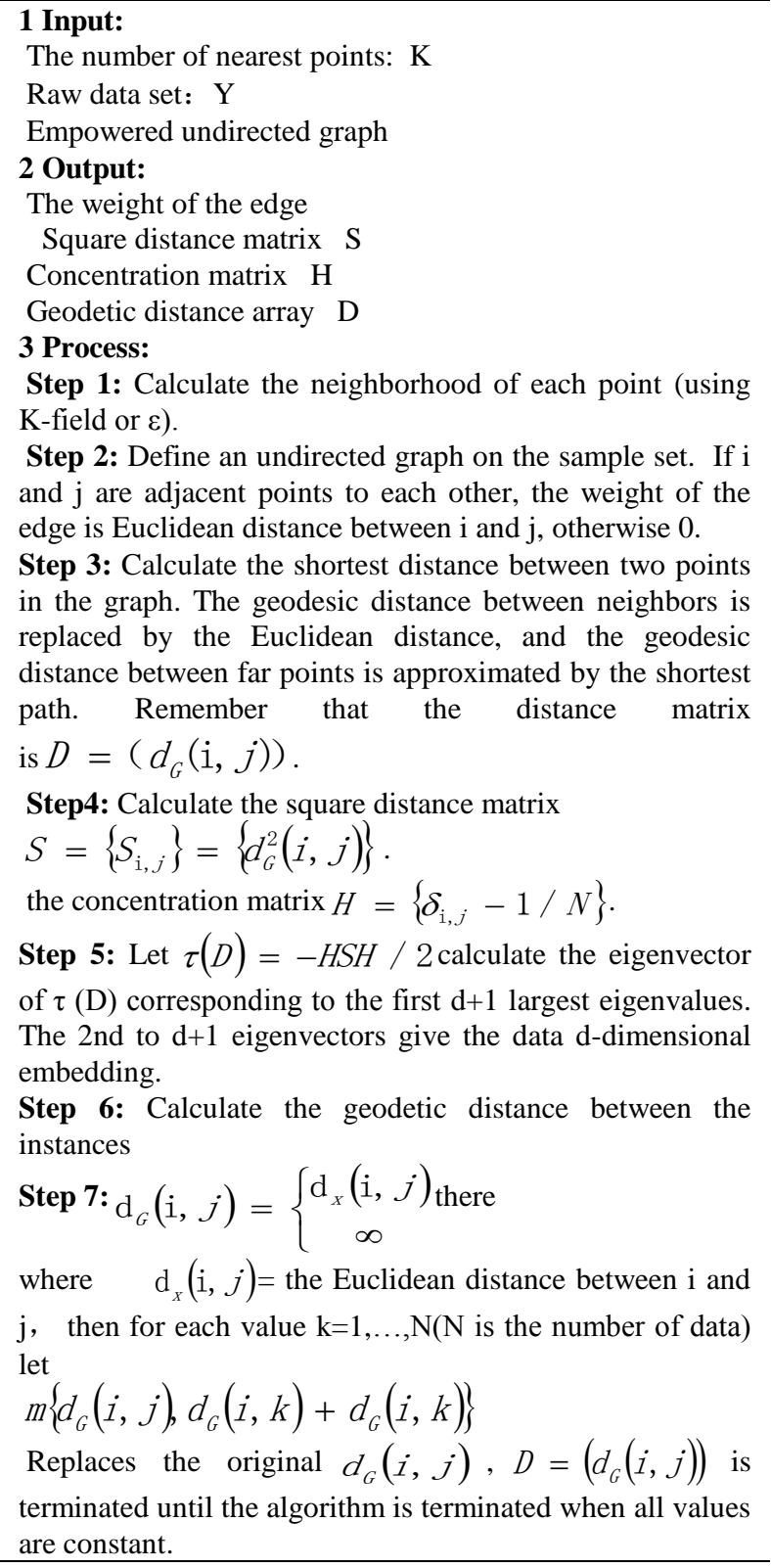

Firstly, the k-nearest neighbor of each input pattern is computed to construct the neighbor graph $\mathrm{M}$. The vertices of the neighbor graph $\mathrm{M}$ represent the input patterns, and in this neighbor graph $\mathrm{M}$ each pattern is associated with its $\mathrm{k}$ nearest neighbor.

The vertices $y_{i}$ and $y_{j}$ are judged whether they need to be connected by the edges $\overline{y_{i} y_{j}}$.In addition to judging by the $\mathrm{k}$ domain method, the judgment of the $\varepsilon$-neighborhood also has the same effect. If it is judged that the Euclidean distance $\left(y_{i}, y_{j}\right)$ between $y_{i}$ and $y_{j}$ is smaller than the given threshold $\varepsilon$, it is judged that $y_{i}$ and $y_{j}$.

\subsection{Isomap-based CL-DT algorithm}

When the above steps are successful, we can only get the geodetic distance between the instance and the instance. According to the obtained geodetic distance, we can use the following rules to determine whether there is an R-relationship between the instance and the instance. If only the geodesic distances of the output instances $Y_{i}$ and $Y_{j}$ are less than or equal to a given distance threshold, we consider R-relation exists between them. Mathematical model can be expressed a

$$
R\left(Y_{i}, Y_{j}\right)=\left(\begin{array}{c}
1 \text { if } D_{G}\left(Y_{i}, Y_{j}\right) \leq D_{\theta} \\
\operatorname{nanif} D_{G}\left(Y_{i}, Y_{j}\right) \geq D_{\theta}
\end{array}\right)
$$

where

$$
\begin{aligned}
& D_{\theta}=\text { geodetic distance threshold } \\
& R\left(Y_{i}, Y_{j}\right)=\text { R-relation between instance } Y_{i} \text { and } Y_{j} \\
& D_{G}\left(Y_{i}, Y_{j}\right)=\text { the geodesic between } Y_{i} \text { and } Y_{j} \text { distance }
\end{aligned}
$$

After determining whether there is an R-relation between an instance and an instance, those that satisfy the condition of the R-relation are considered as co-collocated instances of the candidate. After we determine the R-relation between the instances, we get only two instances of candidate co-collocated mode. Therefore, this paper uses the different attributes of the examples obtained from remote sensing images (eg, soil water content, surface temperature and vegetation coverage, etc.) to identify examples of co-collocated patterns. Based on the above reasons, this paper defines a participation index $\mathfrak{R}$ to determine whether the instance is co-collocated. Different attributes (eg soil moisture content, surface temperature, vegetation cover) are used to determine different attributes based on the location of the isomerism. For this reason, a density ratio, $\mathfrak{R}$ is defined to determine whether instances are Isomap-based co-location patterns. The mathematical model of participation index $\mathfrak{R}$ can be expressed as:

$$
\mathfrak{R}=\frac{\operatorname{AttrN}\left(y_{i}, y_{j}\right)_{\text {similar }}}{\operatorname{Attr} N_{\text {Total }}}
$$

where

$$
\mathfrak{R}=\text { density ratio }
$$

$$
\operatorname{AttrN}\left(y_{i}, y_{j}\right)_{\text {similar }}=\text { the number of }
$$

attributes in $y_{i}$ and $y_{j}$

$$
\operatorname{Attr} N_{\text {Total }}=\text { the total number of attributes }
$$

After calculation, when $\mathfrak{R}$ is greater than or equal to the threshold $\mathfrak{R}_{\theta},\left(y_{i}, y_{j}\right)$ is an Isomap-based co-location pattern. If the density ratio of the candidate of the Isomap-based colocation pattern satisfies the threshold constraint condition, the candidate is an Isomap-based co-location pattern. By parity of reasoning, all Isomap-based co-location patterns will be obtained. The decision rules will be created by translating the decision tree into semantic expressions. Because the Isomapbased Cl-DT algorithm partitions a data space into several distinct disjoint regions via axis parallel surfaces, the top-down search method will be employed to translate individual node into rules in this paper. 


\section{ISOMAP-BASED CL-DT ALGORITHM DECISION TREE INDUCTION}

Mainly includes three steps as follows, first the data is expanded by Isomap, the geodesic distance between the examples is calculated. Second, determine the co-juxtaposition patterns and rules, Third summarize the Isomap-based CL-DT decision tree and decision rules.

\subsection{Calculate geodesic distance}

According to the formula from A to B, we obtain the output instances of instances after Isomap processing and the geodesic matrix $D_{G}$.

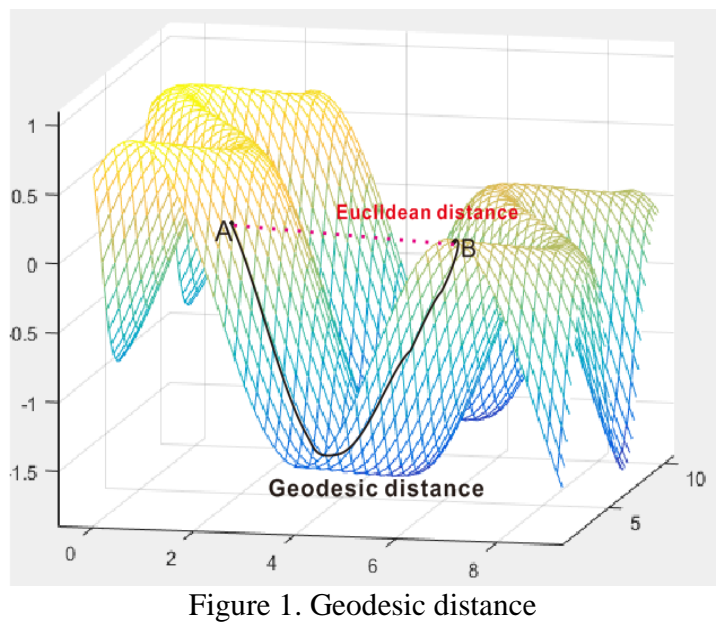

\subsection{Determine the Isomap-based co-collocated modes and rules}

First step is initialized, the instance is expanded and entered and set for each variable and for each variable storage space. Second, Collaborative collocation patterns and rules are determined. First, by formula (1) to determine whether these six instances of R-relationship, there is $i \neq j, \forall i, j=1,2,3,4,5,6,7$.After calculation, only the geodetic distance between the expanded instances $S_{1}$ and $S_{2}$ is smaller than $D_{\theta}$.Therefore, the expanded instances $S_{1}$ and $S_{2}$ are candidate coordination and collocated instances. The participation index of candidate co-collocated instances $S_{1}$ and $S_{2}$ is calculated as follows: since the values in $A_{1}$ and $A_{2}$ of the expanded instances $S_{1}$ and $S_{2}$ satisfy the threshold of the corresponding attribute at the same time, therefore

$$
\mathfrak{R}\left(S_{1}, S_{2}\right)=\frac{\operatorname{AttrN}\left(S_{1}, S_{2}\right)_{\text {similar }}}{\operatorname{Attr} N_{\text {Total }}}=1
$$

Where $\quad S_{1}$ and $S_{2}=$ instances

then expand the instance of $S_{1}$ and $S_{2}$ for the collaborative mode. Next determine whether $S_{1}$ and $S_{2}$ are the same event type.

That is: $S_{l}$ and $S_{2}$ is

$$
\Phi_{i}=\sum_{k=1}^{2}\left(\left\|S_{\mathrm{i}}-A_{\mathrm{m}}\right\|\right)^{2}
$$

If $\Phi_{1}<\Phi_{\theta}, \Phi_{2}<\Phi_{\theta}$, then instances $S_{1}$ and $S_{2}$ are the same event type. Then instances $S_{1}$ and $S_{2}$ are the same event type.

\subsection{Summarize the Isomap-based CL-DT decision tree and decision rules}

(1) The root node that contains all the instances is the current point to be tested.

(2)The use of information gain method to select the best attributes, select the attribute as $A_{l}$.

(3) Use the A attribute to split the root node. Because the curr $A_{l}=F_{l 3}$, the obtained child nodes are already homogeneous, and the owned instance $S_{4} 、 S_{5} 、 S_{6}$ is the same type $C_{3}$. Then use attribute $A_{2}$ to continue to classify the unfinished nodes. When using attribute $A_{2}$ to continue recursive operations on unfinished nodes, the program automatically calls the cooperative collocation rules to determine the coordinated and collocated modes of instances in the nodes, $S_{1}$ and $S_{2}$ are synergetic and set modes. So $S_{l}$ is the same as $S_{2}$.

(4) The program is checked whether the stop criterion is satisfied. For the given case, a decision tree is built using the Isomap-based Cl-DT algorithm.

\section{EXPERIMENT AND ANALYSIS}

\subsection{Data selection:}

In order to verify that the Isomap-based Cl-DT algorithm proposed in this paper extracted bare carbonates and tested in Guilin, Guangxi. It is a typical karst landform with a large number of carbonate rocks on the surface. The non-spatial attributes of the data used include: 35176657 examples of surface soil water content, vegetation coverage, texture and surface temperature, and spatial data with $\mathrm{X} / \mathrm{Y}$ coordinates they make up the database. In order to better extract bare carbonates, we divide the examples into four categories: water, vegetation, arable land and bare carbonate (ec). Their main components were WT> 20, VG> 8, CL <-18, EC <-5. Surface temperature range: $898 \mathrm{~K} \sim 305 \mathrm{~K}$. Spatial properties are $\mathrm{X} / \mathrm{Y}$ coordinates, Progection: Transverse_Mercator; False_Easting: 80000.0000False_Nortjing:0.000000;Central_Meridian:111.00 000 .

\subsection{Experiment process}

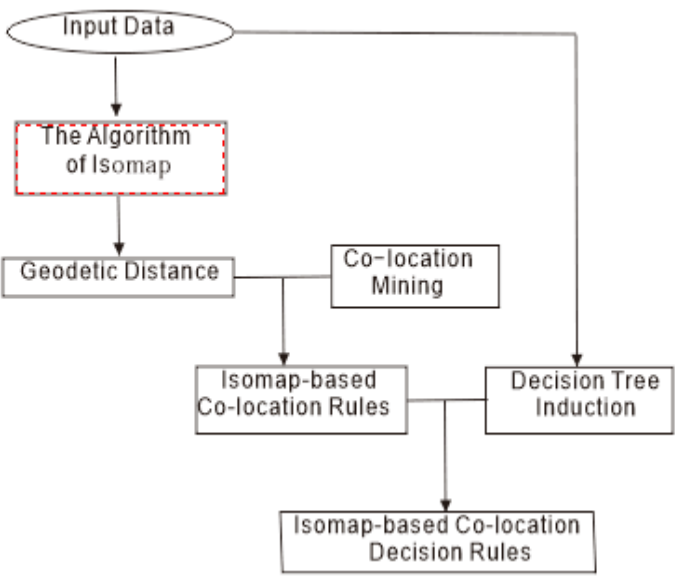

Figure 2. Experiment flowchart

where $\quad \mathrm{i}=1,2$ 
The first is to expand the input data with Isomap algorithm. The geodesic distance that can reflect the relationship between the instances is obtained by calculation. Then the geodesic distance is combined with the collaborative collocated mining algorithm to establish the R-relation between the instances. Collaborative collocation rules guide the construction of decision trees and conclude sets of collaborative decision rules.

\subsection{Experimental results}

In the test area, the same remote sensing image is classified by using the Isomap-based Cl-DT algorithm and the Cl-DT algorithm proposed in this paper. (a) is the Cl-DT algorithm ,(b) is the Isomap-based Cl-DT algorithm. The comparison results are shown in the following image.
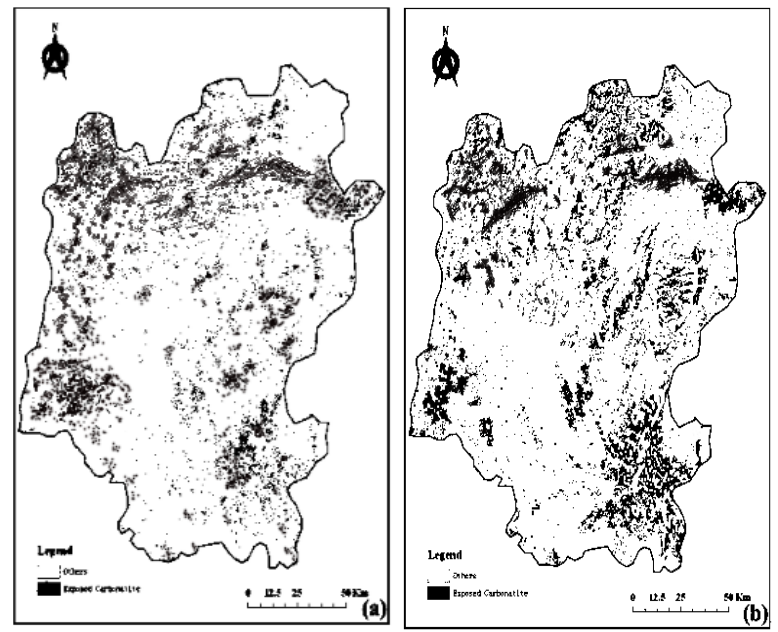

Figure 3. Comparison of remote sensing image classification Results

\begin{tabular}{lll}
\hline \multirow{2}{*}{$\#$} & \multicolumn{2}{c}{ Prod. Acc. } \\
\cline { 2 - 3 } & CL-DT & $\begin{array}{l}\text { Our } \\
\text { method }\end{array}$ \\
\hline Vegetation & $88.37 \%$ & $96.41 \%$ \\
\hline Water & $92.02 \%$ & $94.50 \%$ \\
\hline Exposed Carbonatite & $71.31 \%$ & $84.91 \%$ \\
\hline Habitation & $76.25 \%$ & $94.83 \%$ \\
\hline Cultivated Land & $78.93 \%$ & $86.07 \%$ \\
\hline \multicolumn{2}{c}{ Table 1. Result data comparison }
\end{tabular}

\section{CONCLUSIONS}

The primary contribution of this research is to propose a new method called Isometric Mapping (Isomap)-based Cl-DT. The algorithm, which overcomes the deficiency of the traditional ClDT method that Euclidean distances of instances that are nonlinear distributions in higher space cannot accurately represent the real distances of instances through merging the maximum variance unfolding algorithm with the co-location decision tree. This paper has provided detailed descriptions of algorithms and steps. The overall accuracy of the remote sensing images obtained by the proposed algorithm is $91.5 \%$. The accuracy of the remote sensing images obtained by the $\mathrm{Cl}$ DT algorithm is less than $90 \%$. Compared with the Cl-DT algorithm, the total number of tree nodes and the number of leaf nodes are reduced by $47 \%$. The comparison results show that the $\mathrm{W}$ algorithm can build a decision tree faster and better.

\section{ACKNOWLEDGEMENT}

This paper is financially supported by the National Key Research and Development Program of China under Grant numbers 2016YFB0502500, the National Natural Science of China under Grant numbers 41431179, the State Oceanic Administration under Grant numbers 2014\#58, GuangXi Natural Science Foundation under grant numbers 2015GXNSFDA139032, Guangxi Science \& Technology Development Program under the Contract numbers GuiKeHe 14123001-4, GuangXi Key Laboratory of Spatial Information and Geomatics Program under Grant numbers 151400701, 151400712 and 16380251.

\section{REFERENCES}

G. Zhou, L. Wang, Co-location decision tree for enhancing decision-making of pavement maintenance and rehabilitation. Transportation Research Part C. Emerging Technologies,vol. 21, no. 1, pp. 287-305, 2012.

G. Zhou, Co-location decision tree for enhancing decisionmaking of pavement maintenance and rehabilitation. Ph.D. dissertation, Virginia Tech, 2011.

G. Zhou, R. Zhang. Manifold Learning Co-Location Decision Tree for Remotely Sensed Imagery Classification. Remote Sensing, 2016, 8, 855; doi:10.3390/rs8100855

G. Zhou and R. Li, Accuracy evaluation of ground points from high-resolution satellite imagery IKONOS. Photogrammetry Engineering \& Remote Sensing, vol. 66, no. 9, 2000, pp. 11031112 .

I. H. Witten, E. Frank. Data mining_practical machine learning tools and techniques with Java implementation. Morgan Kaufmann, San Mateo, CA, 2000.

J. R. Quinlan, Induction of decision trees. Machine Learning, vol. 1, pp. 81-106, 1987.

J. R. Quinlan, C4.5: programs for machine learning. Morgan Kaufmann, San Mateo, CA, 1993.

K. Weinberger, L. Saul, Unsupervised learning of image manifolds by semidefinite programming. International Journal of Computer Vision, vol. 70, no. 1, pp. 11-90, 2006.

L. Breiman, J. H. Friedman, R. A. Olshen, and C. J. Stone, Classification and regression trees. California, USA, Wadsworth, 1984.

Osei-Bryson, Kweku-Muata, Post-pruning in decision tree induction using multiple performance measures. Computers \& Operations Research, vol. 34, no. 11, pp. 3331-3345, November 2007.

P. N. Tan, M. Steinbach, and V. Kumar, Introduction to Data Mining. Pearson Addison Wesley,ISBN 0-321-32136-7, 2006.

T. G. Dietterich, Machine learning. Annual Review of Computer Science, vol. 4, 1990. 